\title{
L'accompagnement harmonique dans la musique paysanne roumaine
}

Harmonic accompaniment in Romanian village music

\section{Speranța Rǎdulescu}

Traducteur : Anca Manolescu

\section{OpenEdition}

\section{Journals}

Édition électronique

URL : http://journals.openedition.org/ethnomusicologie/1388

ISSN : 2235-7688

Éditeur

ADEM - Ateliers d'ethnomusicologie

Édition imprimée

Date de publication : 31 octobre 1993

Pagination : 55-67

ISBN : 2-8257-0485-7

ISSN : 1662-372X

Référence électronique

Speranța Rădulescu, "L'accompagnement harmonique dans la musique paysanne roumaine »,

Cahiers d'ethnomusicologie [En ligne], 6 | 1993, mis en ligne le 02 janvier 2012, consulté le 19 avril 2019.

URL : http://journals.openedition.org/ethnomusicologie/1388

Ce document a été généré automatiquement le 19 avril 2019.

Tous droits réservés 


\title{
L'accompagnement harmonique dans la musique paysanne roumaine
}

Harmonic accompaniment in Romanian village music

\author{
Speranța Rădulescu
}

Traduction : Anca Manolescu

\section{NOTE DE L'ÉDITEUR}

Traduit du roumain.

1 Les musicologues roumains rangent les phénomènes musicaux en quatre catégories ou «types syntactiques » : la (M)onodie, la (P)olyphonie, l'(H)omophonie et l'(Hé)térophonie (Niculescu 1969). Ils considèrent que toute musique - écrite ou orale, savante ou populaire - est réductible soit à l'une de ces catégories, soit à leurs superpositions possibles: M/H, Hé/H, M/P, etc. La polyphonie est, de ce point de vue, une syntaxe « espèce » subordonnée au genre plurivocalité, qui inclut Hé, $\mathrm{P}, \mathrm{H}$, tous complémentaires à l'univocalité. J'ignorerai pourtant délibérément cette perspective et j'accorderai au terme de polyphonie le sens de plurivocalité, plus général mais plus proche de son étymologie. La licence que je me permets ne peut pas contrarier mes collègues ethnomusicologues : la musique paysanne roumaine étant fondamentalement monodique, peu d'entre eux ont prêté attention au découpage et à la définition des phénomènes plurivocaux (Alexandru 1980). L'harmonie accompagnatrice est, à leur avis, le produit d'une acculturation tardive qui ne porte pas atteinte à l'essence univocale de la musique et qui, par conséquent, ne les contraint pas à réviser le système terminologique de la discipline.

2 Cette opinion reflète d'ailleurs la réalité dans une large mesure. Le paysan roumain chante et joue par principe monodiquement, que ce soit individuellement ou en groupe. Son univers sonore, qui est pourtant loin d'être strictement linéaire, le prédispose à une perception des simultanéités, car l'ambiance sonore de n'importe quel village est le 
résultat de l'incidence, horizontale et verticale, de différents événements dont les sources de production sont plus ou moins dispersées dans l'espace : grincements de chars, bruits de basse-cour, gazouillements d'oiseaux, heurts d'outils, bribes de conversations et de chansons, etc. Dans les actes rituels, les faits sonores se déroulent parallèlement, leur disposition spatiale leur permettant parfois d'interférer. Naturellement, une concomitance sonore peut être appelée polyphonie uniquement dans le cas d'une production délibérée et conforme à des normes esthétiques précises. Dans la danse, par exemple, musique, cris, mouvements et sons des pas des danseurs forment un ensemble « polyphonique »- le terme est utilisé ici dans son sens figuré, parce qu'il se réfère aussi à des phénomènes situés hors de l'audible - dont le déroulement est contrôlé par le rythme musical. Mais la musique paysanne, même détachée artificiellement du contexte de sa production, met en évidence des rudiments de plurivocalité. Par exemple, les brèves superpositions mélodiques qui surgissent dans le chant antiphonique de certaines pièces cérémonielles (cantiques de Noël, chansons rituelles funéraires) ; les bourdons simples ou doubles des cornemuses; les séquences hétérophoniques qui parsèment les interprétations vocales ou instrumentales de groupes; les sons gutturaux intermittents ou continus, à hauteurs précises ou ambiguës, émis par les joueurs de flûte en même temps que les mélodies; enfin, les sons «nimbés » par plusieurs harmoniques supérieurs distincts de certains instruments (flûte, cor des Alpes, cloches).

Ces germes d'un univers sonore multidimensionnel existent de façon latente depuis des siècles, sans jamais sembler vouloir éclore dans une plurivocalité claire et cohérente. Mais ils préparent une perception auditive verticale et créent indirectement les prémisses de la constitution ultérieure de l'harmonie.

\section{L'ensemble vocal-instrumental rural : le taraf}

L'accompagnement harmonique du chant paysan en milieu rural a surgi il y a un siècle - un siècle et demi à peine, sous la pression de certains facteurs endogènes et exogènes qui, en des moments propices, conjuguent leur action. Son stimulant interne décisif est l'extension sensible de la communauté rurale. Vers la fin du XIXe siècle, les besoins de musique du village réuni lors des fêtes ne peuvent plus être satisfaits par les sonorités discrètes de la guimbarde, de la flûte ou de la cornemuse. Pour y suppléer, les gens recourent à divers artifices. Ils remplacent d'abord les instruments délicats par d'autres, plus pénétrants: la flûte prend la place de la guimbarde; la cornemuse s'impose au détriment de la flûte; le violon ou la clarinette - premières importations du séduisant monde de la ville - écartent peu à peu les précédents. Ensuite, les villageois réclament avec insistance que l'unique instrument mélodique soit doublé par un autre, à l'unisson ou à l'octave. Enfin, ils placent l'instrument soliste ou le petit groupe instrumental à l'endroit le plus avantageux pour la propagation des sons : au milieu des danseurs, au centre d'une grange, sur la ridelle d'un chariot, sous un kiosque, etc.

5 Ces artifices deviennent pourtant assez vite inopérants. Le seul qui soit efficace à long terme est l'augmentation indéfinie du nombre des instruments mélodiques. Mais pendant cette période où les ensembles ruraux multiplient leurs efforts pour fortifier leur potentiel sonore, des endroits tels que la cour du boyard, la foire et la ville - avec lesquels le village multiplie les contacts - offrent déjà une solution attrayante, trop séduisante pour ne pas être essayée : l'ensemble hétérogène et plurivocal, le taraf urbain. 


\section{Le modèle urbain du taraf villageois}

6 Pour tirer au clair la façon dont le taraf urbain s'érige en modèle pour une partie de la musique communautaire rurale, je retracerai rapidement son évolution. Mon incursion sera à la fois lacunaire et prudente, étant donné que les documents qui la jalonnent sont peu nombreux, ambigus et, en tout cas, difficilement interprétables.

7 Les cours des principautés roumaines (la Valachie, la Moldavie et la Transylvanie) se servent, jusqu'au XVIIe siècle, d'ensembles musicaux constitués d'instruments autochtones (flûte et cornemuse) et d'autres empruntés à des cultures différentes (fifre, flûte de Pan, cobza - une sorte de luth -, tympanon) (Bobulescu 1922, 1939). Si l'on en juge d'après les témoignages des chroniqueurs de l'époque, cette musique aurait été, en partie du moins, semblable à la musique paysanne. Il paraît qu'il y avait même des ensembles musicaux qui fonctionnaient comme médiateurs entre le monde rural et celui de la cour (Bobulescu 1922).

8 En Valachie et en Moldavie, le renforcement de la domination ottomane entraîne la création des mehterhane turcs (orchestres d'instruments à vent). Les mehterhane parcouraient les rues des chefs-lieux en jouant; leur musique a donc dû être entendue par le peuple. Au XVIIIe siècle, les mehterhane turcs sont remplacés par des mehterhane roumains, dont les successeurs tardifs seront les fanfares (Gallinescu 1939). A la même époque, la Sublime Porte installe dans les principautés danubiennes des princes étrangers, des Grecs fidèles à l'Empire provenant du Phanar de Constantinople. Les Phanariotes font venir avec eux des formations musicales issues d'une Byzance intensément orientalisée. Cette musique leur est certainement plus proche que celle des Roumains et, par conséquent, plus intelligible et plus captivante. Confiée bientôt aux Tsiganes autochtones, elle se répand vite parmi les boyards et les hauts bourgeois, puis les petits bourgeois, les artisans et les marchands des faubourgs. Les gens simples de la ville l'assimilent en la mélangeant, très probablement, à leur propre musique (dont on ne connaît malheureusement pas grand-chose de précis) et lui confèrent une connotation "populaire». Les habitants des périphéries, citadins de première et de seconde génération, lui impriment un fort caractère rural et la font se répandre plus loin, vers les auberges et les foires fréquentées à la fois par les paysans et les gens de la ville.

9 La musique du taraf des faubourgs du XVIIIe siècle était-elle accompagnée ou non ? C'est possible, mais un tel accompagnement n'a certainement pas pu constituer le point de départ de celui de type tonal-harmonique, développé au siècle suivant. La musique populaire de l'époque phanariote a pourtant, dans son ensemble, de profondes répercussions sur les musiques urbaine et rurale ultérieures : d'une part elle les enrichit par des formules, des timbres et des styles vocaux orientaux qui, en Moldavie et Valachie, sont perceptibles même aujourd'hui ; d'autre part elle familiarise l'oreille des Roumains avec les performances des ensembles instrumentaux et vocaux-instrumentaux hétérogènes.

$10 \mathrm{Au}$ XIXe siècle, la suzeraineté turque s'affaiblit, puis s'éteint. Les élites cultivées se tournent du côté de l'Occident. Les boyards et la grande bourgeoisie commencent à s'habiller, à se comporter et à s'instruire "à l'allemande » ou "à la française ». Des troupes de théâtre lyrique occidentales descendent dans les grandes villes, en proposant des alternatives sonores fraîches à un monde qui s'était trop longtemps cantonné dans un byzantinisme dégénéré et étiolé. En Transylvanie, où le goût pour l'Orient fut toujours 
plus superficiel, la culture occidentale fortifie sa position et ne fait que « rafraîchir son visage ». Princes et nobles poussent leurs musiciens-serfs tsiganes à apprendre la musique de l'Ouest. Même si l'apprentissage est rarement formel et approfondi, il ne tarde pas à porter ses fruits. Les musiciens absorbent, par tous leurs pores, les nouveautés de provenances diverses. Analphabètes du point de vue musical, certains d'entre eux entrent pourtant dans les premiers orchestres philarmoniques nationaux. toute façon un mélange bizarre, mais vigoureux et expansif, de chansonnettes de facture balkanique et de chants et danses paysans; un mélange complété maintenant par des sérénades, quadrilles, valses et romances d'auteur : on se trouve au croisement de l'Orient et de l'Occident, du savant et du populaire. L'européanisation affecte de manière désordonnée ce répertoire, ainsi que le profil de ses mélodies. Le domaine où son action est la moins structurée et la plus lente est celui des accompagnements harmoniques, encore très sommaires et timides.

Le village réagit prudemment et sans hâte à ces changements. Inerte, il a besoin de quelque temps pour comprendre et goûter l'expression musicale plurivocale et tonale, et trouver des modalités pour l'assimiler et la concilier avec des mélodies difficilement réductibles à la tonalité. Pour les fêtes de mariage les plus fastueuses, les paysans riches engageaient déjà depuis longtemps des musiciens professionnels, des lăutari, ou des taraf de la ville. Mais le taraf emprunté et le taraf propre sont deux choses bien différentes : le premier révèle l'altérité, le second reflète l'identité ; celui-là déclenche des changements, celui-ci offre le confort d'une relative stabilité. L'ensemble musical composé de paysans nés et élevés dans le climat spirituel du village surgit lentement et tardivement, à peine vers le début du XXe siècle, en refaisant pas à pas, avec d'inévitables maladresses, le trajet du taraf urbain. Par conséquent, le taraf rural et, implicitement, l'harmonie qu'il est à même de développer progressivement, ne constituent pas de simples "biens échus", mais des acquis sélectifs doublés d'un effort d'adaptation profond et original.

Un des catalyseurs des symbioses culturelles complexes que j'ai évoquées ici est le musicien tsigane, affranchi par la loi au seuil des révolutions de 1848. Pour les Tsiganes des villes (les "serfs princiers»), la libération est quasi formelle, elle ne fait que reconnaître officiellement un état de fait et, éventuellement, lever toute barrière à leur déplacement sur des espaces amples, à la recherche de leur clientèle. Mais les Tsiganes des domaines seigneuriaux et ecclésiastiques, dont l'asservissement avait été vraiment très dur, changent radicalement leur façon de vivre. Ils s'établissent à la périphérie des villages et offrent sans délai leurs services musicaux à leur communauté d'adoption. Ils cherchent en même temps des engagements dans les villages voisins ou plus éloignés, jouent à titre temporaire dans les auberges et les buvettes de villages et même dans les tavernes des faubourgs. Pour plaire aux villageois - qui restent leurs bénéficiaires les plus constants et exigeants - ils s'empressent de remanier leurs performances musicales au goût de ceux-ci.

14 Le rôle des musiciens tsiganes dans la constitution du taraf et dans l'harmonisation paysanne est sans doute important. Je crains pourtant qu'on ait tendance à le surestimer, car il $\mathrm{y}$ a des régions roumaines où leur implication dans la vie musicale du village a été dérisoire ou tardive (Oash, Maramuresh, Bucovine, une partie de la Transylvanie). La nouveauté musicale y est colportée par les paysans eux-mêmes, qui diversifient à tous les niveaux leurs contacts avec le monde extérieur par leurs migrations, toujours plus intenses, rattachées au commerce, au travail saisonnier, à l'armée et à la guerre. 


\section{La crise de la musique communautaire et son dépassement par le recours au taraf}

Le taraf rural est donc la solution permettant de dépasser une crise qui peut être décrite brièvement comme suit.

Au XIX ${ }^{e}$ siècle, le village roumain se dilate, risquant de perdre sa cohésion. La musique se trouve dans une situation de " déficit sonore » qui l'empêche de remplir intégralement sa fonction sociale. La ville, à son tour, s'amplifie et s'occidentalise. Elle intègre massivement des villageois et recourt de plus en plus aux produits et aux services du village. Celui-ci s'habitue à la musique hétéroclite de la ville et s'en empare, même de façon déformée. Les musiciens professionnels, pour la plupart des Tsiganes, facilitent tous les types d'interférences culturelles: entre le village et la ville d'une part, entre divers milieux sociaux-culturels d'autre part. Étant donné que les artifices par lesquels le village essaie d'augmenter le potentiel dynamique de sa musique communautaire se révèlent insuffisants, les Tsiganes autochtones - riches de leur expérience de la musique de cour, de faubourg et de foire acquise antérieurement - composent de petits groupes instrumentaux hétérogènes qu'ils proposent aux villageois. Les paysans acceptent la proposition, confiant désormais l'animation musicale des fêtes à ces formations. Les villages où il n'y a pas de Tsiganes se trouvent devant trois alternatives pour lesquelles ils optent successivement ou au choix : ils conservent pour un certain temps l'ancienne musique monodique, avec tous ses inconvénients; ils engagent des musiciens dans les localités voisines ou dans les villes; ils créent leur propres groupes de musiciens roumains.

\section{Le taraf rural et son accompagnement harmonique}

17 Le taraf se substitue donc à l'instrument vernaculaire qui l'a précédé lors des danses villageoises, des noces, des baptêmes et d'autre fêtes importantes. Dans une première étape, la flûte, la guimbarde ou la cornemuse sont remplacées par un instrument plus vigoureux et pénétrant, d'origine urbaine : le violon, le cobza ou la clarinette. Dans l'étape suivante, le nouvel instrument s'associe avec un ou deux autres, éventuellement de la même catégorie, qui le fortifient par le redoublement à l'unisson ou à l'octave. Ensuite, un des instruments du groupe commence à se spécialiser dans un accompagnement fruste, en accords à deux sons de quarte et de quinte ou en succession lente et quasi-irrégulière : il se transforme ainsi en noyau du futur compartiment second, harmonique, du taraf. Par une mutation décisive, ces accords se métamorphosent en accords complets placés sur la tonique, la dominante ou le deuxième degré de la tonalité : la structure binaire du taraf et le profil tonal de ses accompagnements sont déjà configurés. Le taraf in nuce s'élargit par l'adjonction d'autres instruments qui consolident ses parties soliste et accompagnatrice (le Ţambal, la contrebasse et d'autres). L'accompagnement gagne peu à peu en complexité. A un moment donné, il acquiert la cohérence d'un vrai discours harmonique, avec des syntagmes stéréotypés enchaînés selon une logique propre. Le taraf et le langage harmonique d'accompagnement évoluent donc constamment en étroite interdépendance; et les tâtonnements prolongés qui précèdent chaque étape de leur 
chemin commun prouvent le fait que ni l'un ni l'autre ne sont de simples projections de leurs homologues urbains.

\section{L'harmonisation de la musique du taraf rural « classique »}

18 L'accompagnement harmonique paysan existe, nous l'avons vu, depuis un siècle et demi à peine. Dans cet intervalle, il se transforme rapidement, quoiqu'il reste toujours quelque peu en retrait de celui pratiqué par le taraf urbain. Ses transformations convergent vers l'augmentation de la densité harmonique par la multiplication et la complication des accords et par la diversification des enchaînements d'accords. Les accompagnements concrets deviennent plus riches en événements harmoniques de toutes sortes. Sur la ligne ascendante qui unit les pédales doubles aux discours harmoniques flexibles et compliqués d'aujourd'hui, on peut délimiter une période de "complexité moyenne", située approximativement entre 1930 et 1960, dont je traiterai plus largement par la suite. Cette période - considérée comme " classique " pour le taraf villageois roumain - est d'ailleurs l'espace de convergence de deux autres transformations qui ne visent pas la complexité, mais la spécificité des harmonisations. La première a comme finalité implicite l'institution de certaines différenciations, l'autre tend à les dissoudre. Car, jusqu'aux années trente, le langage harmonique d'accompagnement a développé plusieurs branches, plusieurs idiomes clairement individualisés (Rădulescu 1984 : 62-83). Vers la fin des années cinquante - plus exactement au moment de l'implantation de l'accordéon - les différences entre idiomes ont commencé à pâlir, sans jamais s'effacer complètement. Les idiomes harmoniques, très prononcés dans la période classique, sont liés chacun à une région précise. Ils semblent être déterminés par les particularités des mélodies propres à chaque terroir. En réalité, chacun a pris son contour sous l'influence des ressources techniques d'un instrument accompagnateur précis: cobza, Ţambal, guitare, second violon, alto, groupe d'instruments de fanfare, accordéon). A un certain moment, une région choisit, pour une raison ou pour une autre, un instrument accompagnateur, auquel elle confie la direction de la section d'accompagnement du taraf local. L'accompagnement de cet instrument commence à s'associer constamment à des mélodies du même type, en laissant l'impression qu'il est l'effet de celles-ci. En réalité, l'accompagnement et les mélodies sont compatibles, mais il n'y a pas de relation causale entre eux. De toute façon, les idiomes harmoniques « régionaux » ne diffèrent qu'en des détails; leurs éléments et principes de fonctionnement de base sont communs et constituent le fondement du langage harmonique roumain. 


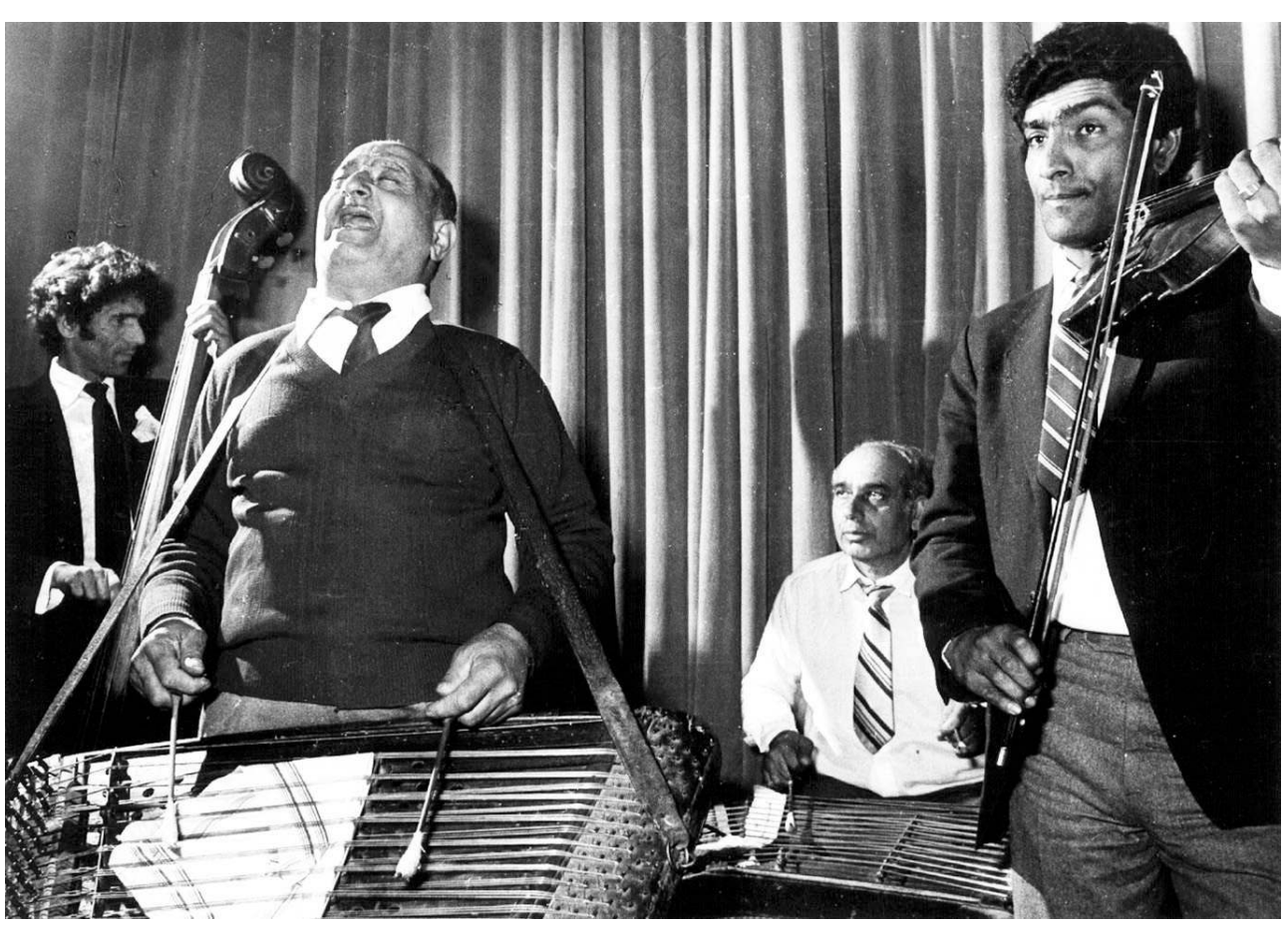

Photo : Dan Dinescu

Ce langage harmonique est explicitement tonal. Le langage mélodique auquel il est subordonné n'est point tributaire de la tonalité ouest-européenne ; mais, sans risquer de modifications importantes, les mélodies qu'il sous-tend permettent, au cours de l'accompagnement, une interprétation tonale provisoire.

Les structures harmoniques élémentaires que je présenterai ici dans l'ordre relatif de leur apparition historique, sont: les accords de quinte et de quarte parfaites (promus par la cobza, la guitare et le second violon); l'accord majeur (constitué autour de la cobza, de la guitare, du second violon et de l'alto en scordatura, mais repris plus tard par tous les autres) ; l'accord mineur (« découvert » par le Țambal mais exclu par la cobza, la guitare et l'alto); l'accord diminué (cultivé tout d'abord par le Ţambal, puis par l'accordéon); l'accord à tierce ambiguë, majeure-mineure. Ce dernier est la seule concession que l'accompagnement tonal fait au contour modal des mélodies. Dans la période d'entredeux-guerres, les accords à trois sons majeurs et diminués s'enrichissent d'une septième, respectivement mineure et diminuée. Toujours à ce moment, les instruments plus agiles (le Trambal et l'accordéon) commencent à agrémenter leurs accords avec des notes mélodiques dissonantes : secondes, quartes et quintes augmentées, septièmes majeures, qui se résolvent respectivement sur la tierce, la quinte, la sixte et l'octave.

Quelle que soit leur qualité (majeure, mineure ou ambiguë), les accords ne peuvent assumer qu'une seule fonction: de tonique, de dominante (du premier, deuxième, troisième ou quatrième degré) ou de sous-dominante. La fonction tonale est toujours plus importante que l'aspect modal des accords.

22 Les enchainements d'accords par quinte, ascendante et surtout descendante, sont les plus fréquents. La connexion sous-dominante - dominante est quasiment absente ; elle essaie d'ailleurs sans succès de s'imposer dans l'accompagnement surtout dans les dernières dix 
années. Une autre relation entre accords, plus audacieuse du point de vue de l'harmonie conventionnelle, apparaît en revanche assez tôt : le déplacement à trois ou quatre quintes ascendantes (donc à la tierce respectivement mineure ou majeure), suivi soit par le retour au ton de départ, de préférence par des descentes successives de quinte en quinte, soit par stabilisation au niveau de la quinte immédiatement inférieure.

Dans le cadre d'une pièce musicale, les modulations tournent autour de la tonalité de base et de sa relative. La distance entre les tonalités les plus éloignées ne dépasse pas quatre quintes, et celle entre les accords excentriques, cinq, six quintes au maximum. De même que les enchaînements entre accords, les modulations sont réalisées de manière diatonique, plus rarement chromatique, et dans certains cas - et seulement dans l'idiome développé par le ţambal - enharmonique (par la réinterprétation enharmonique d'un son de l'accord diminué à septième diminuée).

L'élaboration des discours enharmoniques d'accompagnement se soumettent aux principes suivants :

1. Le principe de la répartition différenciée des voix de l'harmonie par instrument. Les voix de l'harmonie sont distribuées exclusivement aux instruments d'accompagnement. Les instruments solistes doublent accidentellement certaines de ces voix, mais l'ensemble ne compte pas sur leurs redoublements. Le principal instrument d'accompagnement prend en charge l'exposition complète et intégrale du discours harmonique, sans se baser sur les additions des autres instruments. Ceux-ci complètent les sonorités par des redoublements dont l'absence n'altère pas l'intégrité de l'accompagnement.

2. Le principe de l'homogénéité rythmique de la texture d'accompagnement. Les accords sont exposés à travers une formule mélodique et rythmique d'accompagnement nommée tiitură. Le langage harmonique roumain contient approximativement dix de ces formules de base ( Ţiituri) et quelques autres dérivées. L'accompagnement d'une certaine pièce recourt à une seule Ţiitură.

3. Le principe de l'harmonisation économique sur l'horizontale. Tout accord doit soutenir autant de sons que possible de la mélodie accompagnée. Cela signifie que, dans un texte musical donné, les déplacements harmoniques sont assez peu fréquents, les accords ne visant que les point essentiels et stables de la mélodie ; cela signifie aussi qu'une bonne partie des sons de la mélodie sont traités comme notes mélodiques - soit consonantes, soit effectivement dissonantes.

4. Le principe de la prévalence de la fonction tonale sur l'aspect modal des accords. La qualité majeure, mineure, diminuée ou ambiguë des accords est, pour le discours accompagnateur, moins significative que leur fonction tonale. Il est d'ailleurs possible qu'un accord majeur soutienne une mélodie ou un fragment mélodique mineur, sans que le frottement des tierces soit ressenti comme une aspérité. Toutefois, il est presque exclu qu'un accord mineur appuie un segment mélodique d'aspect majeur.

5. Le principe de la corrélation élastique de la mélodie et de l'accompagnement. Les pulsations du discours musical global sont assurées par l'accompagnement, qui est toujours précis du point de vue rythmique. La mélodie est souple, elle peut se permettre des relâchements et des précipitations, à condition que ceux-ci se compensent réciproquement et qu'ils soient interrompus par des moments de parfaite synchronisation. Le degré d'élasticité d'une mélodie dépend du genre auquel elle appartient (par exemple la danse est plus rigoureuse que la chanson, etc.).

6. Le principe de la distribution relativement équilibrée des accords en phrases et sections. La densité horizontale du discours harmonique est relativement réduite et équilibrée. Elle tend toutefois à augmenter légèrement vers la fin des phrases, des périodes ou des sections musicales. Pourtant, la dernière moitié de la phrase cadentielle est obligatoirement 
harmonisée par l'accord de la tonique. La densité harmonique d'un accompagnement ne dépend pas du genre de la mélodie accompagnée, mais de sa configuration particulière.

Conformément à ces principes, le langage musical s'est constitué des syntagmes harmoniques stéréotypés dont les dimensions sont les phrases, les périodes et les sections. Lorsqu'il accompagne une mélodie, un interprète actualise de manière plus ou moins imaginative les clichés les plus adéquats à celle-ci.

\section{Le taraf rural contemporain}

Au cours des vingt ou trente dernières années, le taraf villageois a sensiblement changé d'aspect. Sa composition instrumentale, son répertoire, le style de ses performances se sont remodelés sous l'influence de l'orchestre populaire « officiel » et de l'ensemble de musique légère - formations qui lui ont fait concurrence, soit directement, soit à travers les médias. La communauté rurale - élargie, massivement urbanisée, dénivelée culturellement et peu coercitive - ne s'oppose plus aux infiltrations de la nouveauté exogène. Elle permet aux lăutari de moderniser le taraf à leur gré. Ils en profitent pour remplacer les instruments consacrés jusqu'à maintenant - la guitare, le ţambal, la cobza et même le violon et la contrebasse - par des instruments en vogue - la guitare électrique solo, d'accompagnement et basse, l'orgue électronique, la batterie de jazz. Le taraf s'éloigne de manière flagrante de sa forme classique; il demeure toutefois un taraf, tant que les paysans et les lăutari perçoivent ses déviations par rapport à l'ancien modèle.

\section{L'accompagnement dans le taraf contemporain}

L'accompagnement est aussi entraîné dans des modifications substantielles. Pourtant, ses éléments essentiels - les types de structures d'accords, de fonctions tonales-harmoniques et de formules de figuration (Ţiituri) se conservent quasiment inaltérés. C'est seulement l'accord à tierce ambiguë qui disparait - ou plutôt se purifie, en renonçant à l'ambiguïté de sa condition modale et en adhérant soit au majeur, soit au mineur. Un enchaînement d'accords, jusqu'à maintenant accidentel, essaie de s'imposer avec une agressivité inattendue : sous-dominante/dominante. Son effet est fortement dépersonnalisant, les accompagnement qui l'incluent deviennent conventionnels et « apatrides ». Au fond, c'est seulement maintenant, après sa solide infiltration dans le langage harmonique, que la valeur de son absence antérieure devient évidente.

Les mêmes syntagmes-clichés tonaux-harmoniques régissent l'interprétation des accompagnements. Quelques autres nouveaux clichés, recourant à la relation sousdominante/dominante, sont venus s'y ajouter. La densité horizontale des accompagnements a augmenté d'une manière frappante, par l'insertion de nouveaux accords à fonction de dominante (du premier, second, etc. degré). Elle a augmenté en devenant quasi homogène et en perdant ce jeu subtil de concentrations et raréfactions compensatrices qui lui conféraient sa souplesse. Surabondants, les déplacements harmoniques arrivent à annuler réciproquements leurs effets.

Les musiciens semblent toujours vouloir éviter les agglomérations d'accords excessives. Je ne saurais m'expliquer autrement leur souci spécial - et souvent exprimé - d'éclaircir le discours musical sur d'autres plans, et surtout sur celui des rapports entre mélodie et accompagnement. Ils prennent garde tout d'abord à les rendre compatibles du point de 
vue modal, ce qu'ils réussissent en se débarassant des accords à tierce double ou ambiguë et en renonçant à soutenir les mélodies mineures par des accords majeurs. Deuxièmement, les lăutari qui conduisent la section d'accompagnement des taraf imposent à leurs subordonnés de les suivre promptement et de synchroniser leurs changements harmoniques pour éviter les superpositions accidentelles d'accords différents. Enfin, les musiciens solistes soumettent les mélodies aux rigueurs métrorythmiques de l'accompagnement. Par conséquent, celui-ci devient rigide et perd de sa souplesse. Toutes ces pratiques ont, semble-t-il, une fonction compensatrice: elles soulagent le discours sur la verticale pour contrebalancer sa lourdeur horizontale. Pourtant, la compensation est obtenue aux dépens de l'improvisation et de l'élasticité des phrases. Les lăutari sont très attachés à cette transparence artificielle de leur musique et s'efforcent largement de l'acquérir. Beaucoup d'entre eux s'expriment avec dédain au sujet de leurs confrères vieux-jeu qui continuent à ne pas distinguer le majeur du mineur; ou des solistes incapables de respecter la métrique ou de synchroniser leurs attaques avec les temps forts des formules d'accompagnement. Contrariée par la véhémence inattendue de leurs critiques, j'en provoque quelques-uns à s'expliquer, en montrant perfidement mon admiration pour tel vieil accompagnateur ou soliste. Les musiciens plus âgés sont consternés ; mais les jeunes, sûrs d'eux-mêmes et légèrement arrogants, s'énervent et me contredisent énergiquement. Les arguments qu'ils invoquent sont les performances de certains chefs d'orchestre-vedettes, promus par le disque, la radio et la télévision, de grands hommes, de vrais professionnels (sous-entendu: pas comme vous). Après avoir compris que leurs convictions sont inébranlables, je change de tactique: je propose à un violoniste bon, mais hyperprécis, d'interpréter la même chanson selon deux versions : l'une sévèrement mesurée, et l'autre plus flexible, plus détachée de la pulsation métrique. (Pour me faire comprendre, je lui suggère dans le second cas de demander à son accompagnateur une tiitura rapide, qui prédispose à une interprétation rubato.) Le lăutar se conforme à mon exigence. Puis, après l'échec de son dernier essai, il me dit: «Ce que vous me demandez est très difficile! " C'est difficile, vraiment, parce que l'interprétation exacte est ou est devenue pour lui une seconde nature.

En fin de compte et dans un certain sens, les musiciens ont raffiné leur ouïe. Ils sont devenus sensibles non seulement aux fonctions tonales-harmoniques appropriées aux mélodies, mais aussi à la qualité modale des accords accompagnateurs; plus vigilants en ce qui concerne l'exactitude métrico-rythmique des interprétations; plus conscients des détails de la résultante verticale des performances. Leur goût pour le savant, le complexe et l'impeccable s'est formé non seulement à l'école de la musique populaire diffusée par les mass médias, mais aussi par les contacts avec des chefs d'orchestre, des solistes et des interprètes ordinaires des orchestres populaires répandus à travers le pays. Il leur semble que les harmonisations économiques et un peu négligentes les disqualifient professionnellement; mais ils perdent de vue qu'elles ont une propriété tout-à-fait "savante »: celle de traiter une bonne partie des notes des mélodies comme des sons effectivement dissonants. En fait, les lăutari d'aujourd'hui ont des attitudes différenciées quant aux appogiatures et aux passages dissonnants : ceux qui appartiennent à la mélodie sont considérés comme des impuretés et sont éliminés dans les limites du possible par leur transformation en notes réelles et par leur harmonisation; ceux qui se trouvent dans l'accompagnement sont spécialement appréciés et cultivés assidûment, surtout dans les interstices des phrases musicales, dans les cadences, là où ils risquent moins d'entrer en conflit avec le plan mélodique du discours musical. 
31 L'accompagnement harmonique a subi ses changements les plus évidents au niveau du timbre. Les taraf, et surtout leur section d'accompagnement, ont modifié leur formation instrumentale. Les Ţambal, les altos et les accordéons - qui eux-mêmes ont remplacé les cobza, les guitares ou les altos en scordatura et les seconds violons - ont cédé la place aux guitares électriques et surtout aux orgues électroniques. Mais pour l'instant, les anciens instruments ont échappé à leur complète suppression du taraf: pour les noces, on joue beaucoup dans la rue où il n'y a pas de prises électriques; puis les pannes de courant sont très fréquentes en Roumanie et les gens se voient obligés de tenir en réserve les instruments autonomes se passant d'énergie autre que celle du musicien.

Les substitions instrumentales actuelles, tout comme celles qui les ont précédées d'ailleurs, visent à mon avis non pas tant le rafraîchissement des couleurs sonores que l'augmentation du potentiel dynamique du taraf. La preuve en est que les timbres de l'orgue sont médiocrement exploités par les musiciens, parfois même ignorés. L'obsession des intensités massives - bizarre maintenant, quand les ensembles de lăutari utilisent fréquemment des sonorisations - est très probablement le reflet d'une inertie. D'autre part, il n'est pas exclu que je me trompe en la considérant comme déterminante dans le déclenchement des substitutions instrumentales. Mais quoi qu'il en soit, celles-ci existent et ne tardent pas à se traduire sur le plan de la structure des accompagnements. Car chaque instrument formule son discours sur les coordonnées générales du langage harmonique roumain, mais dans les termes d'un idiome harmonique régional, dont les particularités dépendent de ses ressources techniques et harmoniques. Son remplacement par un autre instrument provoque une déviation de l'accompagnement. Or l'orgue électrique est si riche en possibilités harmoniques qu'il prédispose, plus que tout autre instrument, aux transformations les plus osées. Par son truchement, un lăutar, même médiocre, découvre facilement l'univers plurivocal de toute musique à large diffusion. L'orgue s'est d'ailleurs intégré dans le taraf en amenant avec lui toute une série de clichés d'accompagnement de la musique disco - en vogue maintenant dans les villages roumains. Elle facilite, semble-t-il, un rapprochement entre la musique paysanne et les plus récentes musiques importées de l'Occident. Même les lăutari sont frappés par ce fait. Deux d'entre eux me racontaient qu'ils ont entendu à la discothèque du village des mélodies locales schématisées, devenues presque méconnaissables. Un autre me faisait remarquer la similitude entre l'accompagnement de la musique disco et celui de la musique populaire. Le fait qu'un instrument récemment adopté dans le taraf catalyse l'insertion d'éléments de provenance occidentale n'est pas nouveau. Mais ce qui est surprenant, c'est que les lăutari se désintéressent complètement du potentiel mélodique de l'orgue. On aurait pu s'attendre à ce qu'ils traitent l'instrument comme un accordéon capable de soutenir à la fois le plan harmonique et mélodique des chants ; mais ce n'est pour l'instant pas le cas.

33 L'orgue est un instrument destructif pour la musique de taraf en général et pour son accompagnement harmonique en particulier. Le simple fait que, à l'instar de l'accordéon, et même plus que celui-ci, il s'installe de manière indifférenciée sur tout le territoire du pays en minant les taraf et les idiomes harmoniques régionaux et en instaurant à leur place un langage unique et impersonnel est suffisant pour justifier mon affirmation. Peu nombreux sont les taraf qui le repoussent, peu nombreux les instruments d'accompagnement qui résistent et gardent en même temps leur accompagnement spécifique; comme la fanfare populaire de Moldavie par exemple, dont la force de pénétration défie tout instrument concurrent, toute sonorisation. Cette fanfare, une 
apparition assez tardive dans la musique paysanne, aux apparences novatrices agressives, s'est transformée paradoxalement en une redoute traditionnaliste. En tout cas, son accompagnement harmonique est très conservateur car, élaboré en groupe, il se prête fort mal à l'innovation.

\section{BIBLIOGRAPHIE}

ALEXANDRU Tiberiu, 1980, « Armonie si polifonie in cîntecul popular românesc », in : Folcloristică, organologie, muzicologie. Studii. Bucureşti : Editura Muzicală : 22-116.

BENTOIU Pascal, 1965, « Citeva aspecte ale armoniei în muzica de populară din Ardeal », in : Studii de muzicologie I. Bucureşti : Bucureşti : Editura Muzicală : 147-214.

BOBULESCU Constantin, 1922, Lăutarii nostri. Din trecutul lor. SchiŢă asupra istoriei muzicei noastre na Ţionale corale precum şi asupra altor feluri de muzici. București : Tip. « NaŢională » Jean Ionescu \& Co.

BOBULESCU Constantin, 1939, « Muzica în Muntenia. Lăutarii în manuscrise, Ţipărituri şi vechile zugrăveli ale bisericilor din Muntenia ", in : Muzica românească de azi, éd. par P. NiŢulescu. Bucureşti, pp. 604-669.

BOUET Jacques, 1973, « Les violonistes et l'exécution violonistique dans le milieu de tradition orale roumaine », in : Studii de muzicologie 9. București : Editura Muzicală : 351-440.

BOUET Jacques, 1984, «Fioritures et accompagnements dans les faubourgs et les villages », in: Dialogue. Revue d'études roumaines et des traditions orales méditerranéennes 12/13. Montpellier : Université Paul Valéry : 63-77.

GALINESCU Gavriil, 1939, « Muzica în Moldova », in : Muzica românească de azi, éd. par P. NiŢulescu. Bucureşti, pp. 671-728.

HABENICHT Gottfried, 1964, « Accompaniamentul tarafurilor năsăudene », in : Revista de etnografie şi folclor 9. Bucureşti : Editura Academiei : 339-347.

LORTAT-JACOB Bernard, 1984, «Le métier de lăutar », in : Dialogue. Revue d'études roumaines et des traditions orales méditerranéennes 12/13. Montpellier : Université Paul Valéry : 13-36.

NICULESCU ştefan, 1969, «Eterofonia », in : Studii de muzicologie 5. București : Editura Muzicală : 65-74.

NICULESCU ştefan, 1984, Taraful şi acompaniamentul armonic în muzica de joc. București : Editura Muzicală.

\section{RÉSUMÉS}

The author attempts to correlate two phenomena which have influenced collective musicmaking in the Romanian countryside: the crisis it has undergone under the impact of, inter alia, the marked growth of the rural communities, requiring a louder musical sound; and the possible 
solution offered by a rural ensemble modeled on the urban gipsy ensemble called taraf whose main characteristic is its plurivocality. The author focusses on the historical aspects of this musical change and analyses the harmonic language developed by the rural taraf.

\section{AUTEURS}

\section{SPERANTA RĂDULESCU}

Speranţa Rădulescu est née en 1949. Après avoir suivi des études de composition musicale au Conservatoire de Bucarest, elle devient chercheur à l'Institut d'ethnographie et de folklore - à l'époque appelé Institut de recherches ethnographiques et dialectologiques - de 1973 à 1990, où elle est nommée responsable du Département de Musique et chorégraphie populaire en 1980. Depuis 1990, elle dirige le Département d'anthropologie culturelle du Musée du paysan roumain de Bucarest. Parmi ses publications, on peut mentionner Taraful şi acompaniamentul armonic în muzica de joc (Le taraf et l'accompagnement harmonique dans la musique de danse) (1984), et Cîntecul. Tipologie muzicală. vol. 1 (La chanson. Typologie musicale. vol. 1) (1990), ainsi que deux collections de disques : The Traditional Folk Music Band, série de 6 LP (Electrecord, dès 1984), et Roumanie : musique de villages, coffret de 3 CD (VDE, collection AIMP, 1988). Elle organise aussi occasionnellement des concerts de musique traditionnelle. 